\title{
Teoria da Mente em Familiares de Autistas: Uma Revisão da Literatura
}

\author{
Aline Abreu e Andrade * Maycoln Lê̂ni Martins Teodoro \\ Universidade Federal de Minas Gerais, Minas Gerais, Brasil \\ Walter Camargos Junior \\ Fundação Hospitalar do Estado de Minas Gerais (FHEMIG), Minas Gerais, Brasil
}

\begin{abstract}
RESUMO
Teoria da mente consiste na habilidade de compreensão das pessoas sobre seus próprios estados mentais e dos outros. Pessoas com autismo possuem déficit nesta habilidade. O objetivo deste estudo foi realizar uma revisão sistemática da literatura sobre os estudos de teoria da mente realizados com familiares de pessoas com transtornos do espectro autístico. Utilizou-se as bases de dados BVS Medline, Web of Science, Academic Search Premier e Psy Articles na busca dos artigos. Foram selecionados nove artigos e os resultados indicaram inconclusibilidade dos dados sobre a existência de déficits de teoria da mente em familiares de pessoas com transtornos do espectro autístico. Desta forma, são apontados possíveis estudos futuros que possibilitem lançar luz sobre o tema.
\end{abstract}

Palavras-chave: teoria da mente; autismo; pais; irmãos.

\begin{abstract}
Theory of Mind in Autistic Family: A Literature Review

Theory of mind is the people ability to understand their own mental states and the others. People with autism have significant deficit in this ability. The objective of this study was to conduct a systematic literature review about theory of mind studies conducted with relatives of people with autistic spectrum disorders. We used as databases BVS Medline, Web of Science, Academic Search Premier and Psy Articles in search of articles. Nine articles were selected and the results indicated inconclusive data on the existence of theory of mind deficits in relatives of people with autistic spectrum disorders. Thus, future studies are aimed to shed light on the topic.
\end{abstract}

Keywords: theory of mind; autism; parents; siblings.

O conceito de teoria da mente (ToM) surgiu no fim da década de 70 a partir de experimentos sobre cognição animal (Premack \& Woodruff, 1978). Este conceito vem sendo amplamente estudados hoje por etologistas, filósofos da mente, psicólogos cognitivistas e do desenvolvimento e especialistas em autismo (Roazzi \& Santana, 2008).

Teoria da mente é definida por Premack e Woodruff (1978) como a capacidade de atribuir estados mentais para si mesmo e para os outros, desenvolvendo uma medida daquilo que eles pensam, sentem, desejam, acreditam, duvidam. É, assim, um sistema de inferências que permite comparações entre o mundo pessoal e o mundo das outras pessoas.
Embora teoria da mente e empatia possam ser considerados como construtos semelhantes, destaca-se que eles apresentam períodos de desenvolvimento e substratos neurais distintos. Enquanto a rede de empatia é considerada funcional desde a primeira infância (De Vignemont \& Singer, 2006; Gallese, 2003; Lamm, Batson, \& Decety, 2007; Singer, 2006) e, possivelmente, baseada em um sistema humano de neurônios-espelho (Gallese, 2003; Shamay-Tsoory, Aharon-Peretz, \& Perry, 2009), a rede da teoria da mente demonstra um desenvolvimento que se prolonga ao longo da infância e envolve a junção têmporo-parietal, córtex parietal medial e córtex pré-frontal medial (Frith \& Frith, 2003). Desta forma, cabe esclarecer a

\footnotetext{
*Endereço para correspondência: Aline Abreu e Andrade - aline_abreu_andrade@yahoo.com.br
} 
distinção entre os dois conceitos. Enquanto a empatia se refere à capacidade de responsividade emocional a outras pessoas, a teoria da mente se constitui na percepção cognitiva a respeito das intenções e crenças dos outros, inclusive as falsas crenças (Ford, Lobao, Macaulay, \& Herdman, 2011).

A avaliação do desenvolvimento de teoria da mente em crianças é comumente feita pela "tarefa de falsa crença". O paradigma desta tarefa se consolidou na década de 70, a partir do trabalho de Dennett (1978), que postulou que a atribuição de uma teoria da mente a um indivíduo só é possível se ele demonstra uma compreensão da tarefa de crença falsa, isto é, de que uma crença conflita com a realidade (Jou \& Sperb, 1999).

A tarefa de falsa crença padrão, utilizada na maioria dos estudos, foi elaborada por Wimmer e Perner (1983) e é denominada tarefa de Maxi. É relatada para a criança a seguinte situação: "Maxi está ajudando sua mãe a guardar as compras na cozinha e coloca o chocolate no armário verde. Maxi lembra exatamente onde colocou o chocolate e, por isto, ele pode voltar mais tarde e pegar um pouco. Então ele vai ao pátio. $\mathrm{Na}$ sua ausência a mãe pega o chocolate do armário verde e usa um pouco na torta. Depois ela coloca o chocolate dentro do armário azul, e não do verde. Então ela sai e Maxi volta do pátio com fome. Onde Maxi vai procurar pelo chocolate?". Diz-se que a pessoa já possui uma teoria da mente desenvolvida quando ela responde que "Maxi irá procurar o chocolate no armário verde", uma vez que ele não viu e não sabe que sua mãe trocou o chocolate de lugar. Por outro lado, diz-se que a criança não possui uma teoria da mente desenvolvida quando responde que "Maxi irá procurar o chocolate no armário azul".

Uma variação deste teste é chamada tarefa SallyAnne, na qual as crianças também são convidadas a prever onde um personagem vai procurar primeiro por um objeto que foi movido para um novo local em sua ausência (Baron-Cohen, Leslie, \& Frith, 1985). Vários estudos confirmam que crianças a partir da idade de quatro a seis anos apresentam resposta correta à tarefa de falsa crença (para uma revisão sobre o tema, ver Wellman, Cross, \& Watson, 2001).

Entretanto, crianças com autismo apresentam resultado abaixo do esperado em tarefas que avaliam teoria da mente, déficit amplamente documentado pela literatura (Best, Moffat, Power, Owens, \& Johnstone, 2008; Pellicano, Maybery, Durkin, \& Maley 2006). O transtorno autista é uma síndrome comportamental caracterizada por déficits significativos em três áreas: 1) comprometimento qualitativo da intera- ção social; 2) comprometimento da comunicação e; 3) padrões restritos e repetitivos de comportamento, interesses e atividades com início antes dos três anos de idade e passível de diagnóstico em torno dos 18 meses de idade (APA, 2002).

Em termos diagnósticos, há uma tendência atual na literatura em definir o autismo através de uma abordagem dimensional, em contraposição à categórica. Assim, mais do que um fenômeno "tudo ou nada", o transtorno autista é considerado como o extremo de um continuum de traços autísticos (Scheeren \& Stauder, 2008). A noção de transtorno espectral questiona a existência de várias entidades psiquiátricas distintas em favor de um modelo de continuum de características clínicas, no qual os indivíduos são afetados em diferentes graus (Bolte \& Poustka, 2006), ao qual se dá o nome de transtornos do espectro do autismo (TEA).

Os transtornos do espectro do autismo possuem prevalência de $2,64 \%$ da população e sua ocorrência entre homens e mulheres é de 2.5:1(Kim et al., 2011). A consideração sobre a sua prevalência, bem como sobre a gravidade do transtorno e grau de incapacidade associado ao seu curso reforçam a necessidade de pesquisas sobre o tema.

Sabe-se que as evidências de herdabilidade no autismo se encontram em torno de $90 \%$ (Kelemenova \& Ostatnikova, 2009). Observa-se, assim, um maior risco de ocorrência do transtorno entre irmãos de indivíduos portadores, bem como maior incidência de dificuldades relacionadas ao desenvolvimento (APA, 2002). Há também, nos estudos atuais, crescentes evidências sobre a existência de alterações subsindrômicas em familiares de autistas, ou seja, a presença de características de autismo que não chegam a preencher critérios diagnósticos para se constituir no transtorno propriamente dito, o que sugere a existência de um fenótipo ampliado do autismo (FAA) (Kamp-Becker et al., 2009).

Uma vez que o comprometimento qualitativo da interação social é característico do transtorno autista e que os déficits no componente de cognição social parecem explicar muitos sintomas do transtorno, hipotetiza-se sobre a presença de alterações sub-sindrômicas neste nível em familiares de pessoas com TEA. Estes déficits na cognição social têm sido investigados através de pesquisas de teoria da mente, que é considerada um possível endofenótipo para o autismo. Endofenótipos se constituem em fatores de risco que vão além do simples comportamento observável, que podem ser investigados a partir de medidas bioquímicas, neurofisiológicas, neuroanatômicas e, neuropsicológi- 
cas (Mercadante, Gaag, \& Schwartzman, 2006) e possuem uma maior correlação com os fatores genéticos do que com os comportamentos característicos da síndrome.

Desta forma, o objetivo do presente artigo consiste na realização de uma revisão sistemática da literatura sobre o funcionamento da Teoria da Mente em familiares de pessoas com transtornos do espectro do autismo. A elucidação dos estudos já existentes pode propiciar uma visão abrangente sobre o tema em questão, bem como auxiliar no delineamento de rumos futuros de pesquisa.

\section{MÉTODO}

Foram consultadas as bases de dados BVS Medline, Web of Science, Academic Search Premier e Psy Articles (APA) para a seleção dos artigos. A busca foi realizada em Janeiro de 2013 e se restringiu a publicações em português, inglês ou espanhol disponíveis nas bases de dados. Foram escolhidos como descritores "autism" e "theory of mind" conjugados a "family", "parent", "parents" "relative", "relatives", "mother", "father", "sibling" ou "siblings". A seleção destes termos se baseou nas palavras-chave utilizadas em artigos examinados previamente.
Consideraram-se como critérios para inclusão do artigo na revisão: 1) a amostra em estudo ser constituída por pais ou irmão de pessoas com transtornos do espectro do autismo (TEA); 2) o uso de método quantitativo com a presença de um grupo de comparação e; 3) o estudo possuir como um dos seus objetivos o estudo da teoria da mente. A escolha de estudos empíricos comparativos como critério de seleção dos artigos teve como intuito o enfoque em estudos com delineamento que demonstrem maior confiabilidade, generalidade e replicabilidade. Realizou-se a leitura do resumo de todos os artigos encontrados, de modo a avaliar a sua adequação aos critérios de inclusão.

Os artigos selecionados foram lidos na íntegra e analisados e separados segundo os seguintes tópicos: Amostra, Instrumentos de Coleta de Dados e Resultados. Por fim, os estudos foram agrupados em blocos de acordo com as semelhanças encontradas.

\section{RESULTADOS}

Foram encontrados, ao todo, 252 artigos a partir dos descritores selecionados, já retirados os repetidos. A partir da leitura dos resumos dos mesmos foi realizada a seleção, de acordo com os critérios estabelecidos na Figura 1.

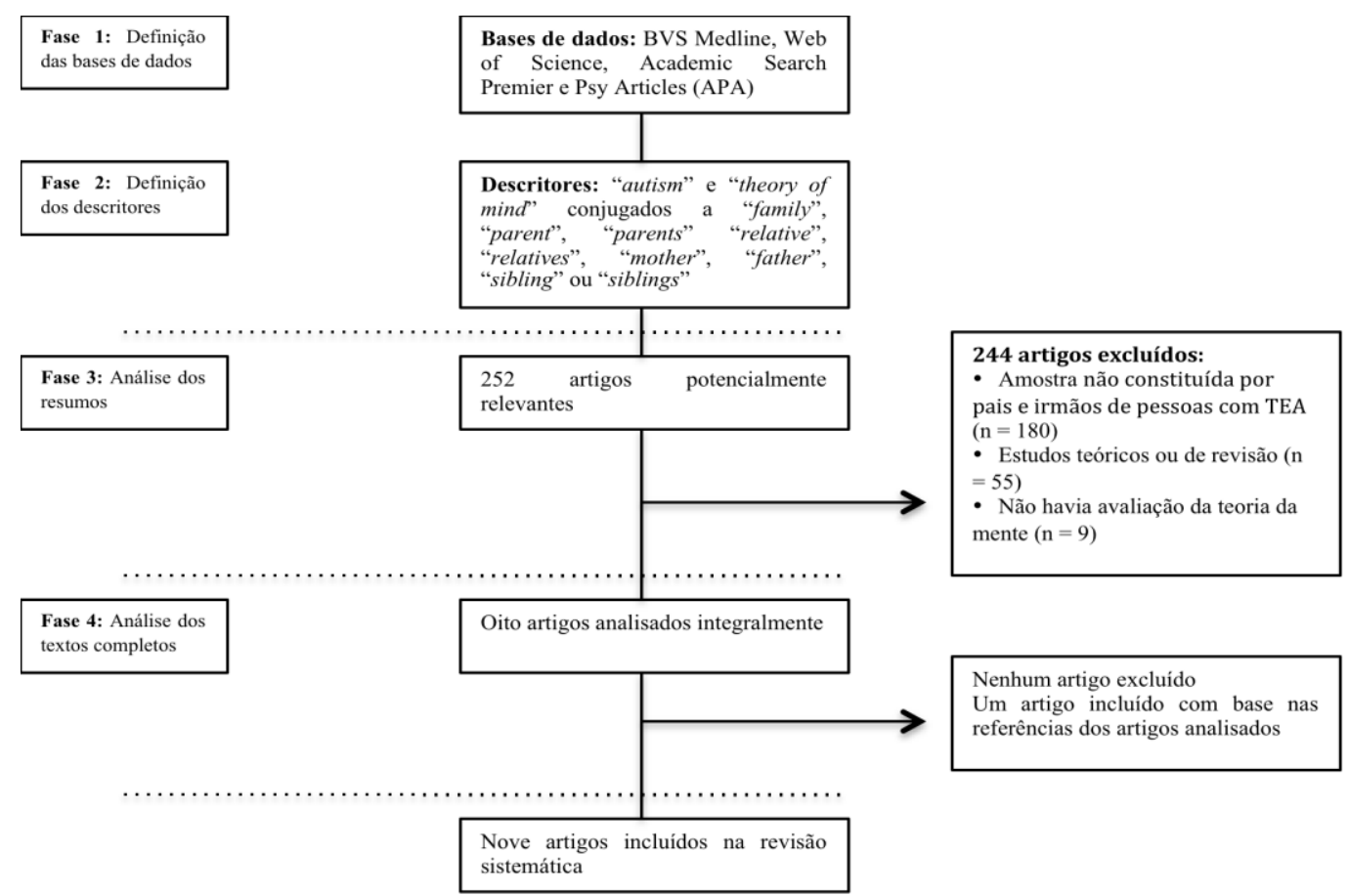

Figura 1. Esquema representativo dos procedimentos de seleção dos artigos. 
Dos 252 artigos encontrados, 180 não possuíam amostra constituída por pais e irmãos de pessoas com TEA. Estes estudos avaliavam crianças, adolescentes ou adultos com diferentes condições (autismo, síndrome de Asperger, anorexia nervosa, esquizofrenia, retardo mental, transtorno de déficit de atenção e hiperatividade, trantornos disruptivos, transtornos do desenvolvimento da linguagem, síndrome de Williams, síndrome do X-frágil e surdez). Como segundo critério, foram retirados 55 estudos que não utilizavam métodos quantitativos; eram artigos teóricos ou de revisão sobre autismo. Por fim, foram excluídos nove artigos que não possuíam como um de seus objetivos o estudo da teoria da mente.

Os oito artigos restantes foram incluídos na revisão. A partir das referências de Gokcen, Bora, Erermis, Kesikci e Aydin (2009) foi encontrado mais um artigo que preenchia os critérios de inclusão (Losh \& Piven, 2007).

Dos nove artigos encontrados sobre teoria da mente em familiares de pessoas com TEA, três enfocaram a avaliação de irmãos (Dorris, Espie, Knott, \& Salt, 2004; Ozonoff, Rogers, Farnham, \& Pennington, 1993; Shaked, Gamliel, \&Yirmiya, 2006), quatro avaliaram os pais (Baron-Cohen \& Hammer, 1997; Baron-Cohen, Ring, Chitnis, Wheelwright, Gregory, Williams, Brammer e Bullmore, 2006; Gokcen et al., 2009; Losh \& Piven, 2007), um investigou conjuntamente pais e irmãos de pessoas com TEA (Nydén, Hagberg, Goussé, \& Rastam, 2011) e um abordou crianças com TEA e seus pais, distinguindo entre pais e mães (Shimoni, Weizman, Yoran, \& Raviv, 2012). Tais estudos serão apresentados a seguir, visando fornecer um panorama geral das pesquisas sobre o tema em questão.

\section{Estudos de teoria da mente com irmãos de pessoas com transtornos do espectro do autismo}

Ozonoff et al. (1993) compararam as habilidades de teoria da mente em irmãos de indivíduos com autismo de alto funcionamento e irmãos de pessoas com transtornos de aprendizagem (foram incluídos transtorno de déficit de atenção e hiperatividade, dislexia, transtorno da linguagem expressiva, e outros transtornos de aprendizagem não especificados). Para tal, foram selecionados 18 irmãos de autistas de alto funcionamento e 18 irmãos do grupo comparação com problema de aprendizagem, com idade entre oito e 18 anos. Os instrumentos utilizados para avaliação da teoria da mente foram o Second Order Belief Attribu- tion Task (Baron-Cohen, 1989), o Fox and Grapes Task e o Apple-Dog Task (Flavell, Botkin, Fry, Wright, \& Jarvis, 1968). Os resultados encontrados no estudo não apontaram diferenças significativas entre os grupos na habilidade de teoria da mente, em nenhum dos três testes utilizados. A adequação destes instrumentos ao estudo também foi investigada. Para tal foram realizadas análises post hoc dos instrumentos empregados na pesquisa, que indicaram que nenhuma das tarefas utilizadas apresentava sensibilidade para discriminar déficits de ToM em familiares. Diante disto, sugeriu-se o desenvolvimento de tarefas de Teoria da Mente mais discriminativas e mais elementares para avaliação de amostras de familiares, uma vez que as existentes foram consideradas demasiadamente complexas, na medida em que requisitavam vários processos cognitivos (Ozonoff et al., 1993).

Já no estudo de Dorris et al. (2004) o objetivo foi examinar as diferenças entre irmãos de crianças diagnosticadas com síndrome de Asperger e um grupo de crianças com desenvolvimento normal, no que se refere à habilidade de ToM. Nesta pesquisa foram selecionados 27 sujeitos de cada grupo. O tamanho desta amostra foi definido através do cálculo de poder amostral, com base nos dados do estudo de BaronCohen e Hammer (1997), uma vez que o estudo de Dorris et al. (2004) se propunha a ser tanto uma replicação quanto uma extensão dos achados desses autores. O instrumento utilizado foi o Eyes Test, publicado por Baron-Cohen, Wheelwright, Stone e Rutherford (1999) e modificado por Baron-Cohen, Wheelwright, Hill, Raste e Plumb (2001). Os resultados deste estudo trouxeram evidências divergentes em relação ao estudo de Ozonoff et al. (1993) sugerindo que irmãos de crianças com síndrome de Asperger demonstravam um desempenho pior em termos de cognição social, se comparados a crianças com desenvolvimento típico. Cabe destacar as diferenças entre os estudos, em termos do tamanho da amostra, grupo de comparação utilizado e instrumentos disponíveis para avaliação de teoria da mente.

Em Shaked et al. (2006) a amostra possuía 24 sujeitos em cada grupo, sendo um dos grupos constituído de irmãos de crianças com autismo e o outro de irmãos de crianças com desenvolvimento típico. $\mathrm{O}$ objetivo desta pesquisa era também explorar se as habilidades de ToM estão prejudicadas em irmãos de pessoas com autismo. Foram utilizadas a False Belief Task (Baron-Cohen et al., 1985) e a Strange Stories Task (três histórias) (Happé, 1994), tendo em vista que o uso de mais de uma tarefa resulta em maior 
confiabilidade na avaliação de habilidades de Teoria da Mente. Os resultados corroboraram os achados de Ozonoff et al. (1993), sugerindo a inexistência de diferenças significativas entre os dois grupos em todas as tarefas que avaliam teoria da mente. De acordo com os autores, este resultado aponta para uma maior resiliência por parte dos irmãos de pessoas com autismo, no que se refere a esta característica em particular.

$\mathrm{O}$ estudo mais recente dentre os encontrados a realizar a avaliação de Teoria da Mente em irmãos de pessoas com TEA (Nydén et al., 2011) possui como distintivo o fato de avaliar conjuntamente sujeitos autistas, pais e irmãos em uma só pesquisa. Assim, a amostra foi constituída de 86 membros, dentre pais, mães e irmãos de pessoas com autismo, de 18 famílias com ao menos dois casos de autismo, sendo realizada comparação entre os grupos. O estudo teve como objetivo examinar o endofenótipo neurocognitivo do autismo, em famílias com múltipla incidência do transtorno. A Teoria da Mente foi avaliada com o uso do Cartoon Explanation Tasks (non-mental e mental) (Gallagher, Happé, Brunswick, Fletcher, Frith, \& Frith, 2000). O instrumento foi aplicado em pais, mães e irmãos de sujeitos com transtorno autístico. Como resultados, também não foram encontrados déficits específicos na execução da tarefa de Teoria da Mente em irmãos. Os autores sugerem, de acordo com Ozonoff et al. (1993) que, sendo a Teoria da Mente uma qualidade sutil, difícil de ser avaliada, é possível que o instrumento utilizado não seja suficientemente sensível para a detecção de déficits desta habilidade.

\section{Estudos de teoria da mente com pais de pessoas com transtornos do espectro do autismo}

O primeiro estudo de avaliação da teoria da mente em pais de pessoas com TEA encontrado tinha como objetivo investigar se pais de crianças com síndrome de Asperger apresentavam manifestações brandas das anomalias cognitivas observadas nas crianças (dentre elas, déficit de teoria da mente) (Baron-Cohen \& Hammer, 1997). Para tal foram avaliados 60 pais biológicos, sendo 30 de crianças com síndrome de Asperger e 30 de crianças sem síndrome de Asperger. Baron-Cohen e Hammer utilizaram em seu estudo a versão do Eyes Test publicada em 1997 (BaronCohen, Jolliffe, Mortimore, \& Robertson, 1997), encontrando como resultado uma menor acurácia de pais de crianças com síndrome de Asperger na interpretação de estados mentais a partir de fotografias da região dos olhos (Eyes Test). Todos os estudos encontrados na presente revisão que avaliam Teoria da Mente em pais de pessoas com TEA utilizaram alguma versão do instrumento Eyes Test.

Ainda utilizando amostra de pais de crianças com síndrome de Asperger, Baron-Cohen et al. (2006) conduziram um novo estudo com o objetivo de testar se eles mostravam atividade cerebral atípica durante a execução de uma tarefa de ToM. Nesta pesquisa foi utilizada uma amostra de 12 pais de crianças com síndrome de Asperger e 12 pais de crianças sem síndrome de Asperger. O Eyes Test foi o único instrumento utilizado para avaliação de ToM. Os autores citam em seu estudo duas referências do teste; a versão de 1997 (Baron-Cohen et al., 1997) e a de 2001 (Baron-Cohen et al., 2001), não sendo possível identificar qual foi utilizada no estudo. Em consonância com Baron-Cohen e Hammer (1997), Baron-Cohen et al. (2006) observaram a existência de diferenças entre os grupos avaliados. Os autores identificaram que familiares de crianças sem síndrome de Asperger apresentavam maior ativação no giro temporal medial e no giro frontal inferior durante a aplicação do Eyes Task, se comparados aos pais de crianças com Asperger. Apesar disto, não foi evidenciada diferença significativa em termos de desempenho dos participantes na tarefa.

Já na pesquisa de Losh e Piven (2007) o objetivo foi relacionar as características comportamentais do fenótipo ampliado do autismo (FAA) ao desempenho em medidas de cognição social em pais. O FAA é definido como a manifestação de características autísticas brandas nos familiares de pessoas com autismo, insuficientes para se constituir em um diagnóstico (Scheeren \& Stauder, 2008). Com este intuito, a amostra foi constituída por 48 pais de indivíduos com autismo e 22 pais de indivíduos com desenvolvimento típico ou com síndrome de Down. Foi utilizado como instrumento de avaliação de ToM o Eyes Test, versão de 2001 (Baron-Cohen et al., 2001). Para os propósitos do estudo, foi aplicado também o questionário Modified Personality Assessment Schedule Revised (MPAS-R) (Piven et al., 1994), que avalia características do fenótipo ampliado do autismo (FAA) em pais. O questionário é constituído por três subescalas de avaliação, sendo 1) personalidade aloof (distante, indiferente), que remete à falta de interesse ou prazer na interação social; 2) personalidade rígida, que indica pouco interesse por mudanças ou dificuldade de adaptação a mudanças e; 3) linguagem pragmática, que sugere deficits nos aspectos sociais da linguagem, resultando em dificuldades de comunicação eficaz ou na realização de uma conversa fluida e com reciproci- 
dade (Hurley, Losh, Parlier, Reznick, \& Piven, 2007). Entretanto, neste estudo foram investigadas apenas duas dimensões do FAA: personalidade rígida e aloof. Os dados encontrados sugeriram que a capacidade de cognição social se mostrava intacta em pais de crianças com autismo, no geral. Somente no subgrupo com personalidade aloof foram observados déficits de cognição social. Os participantes que apresentaram a característica de rigidez no FAA não apresentaram déficits e o subgrupo com déficits de linguagem pragmática não foi avaliado (Losh \& Piven, 2007).

Gokcen et al. (2009), por sua vez, visava investigar o potencial dos déficits de cognição social e função executiva enquanto possíveis endofenótipos do autismo. Para tal, o autor utilizou uma distinção entre duas categorias de habilidades de teoria da mente: 1) de decodificação, que se refere à capacidade de identificar os estados mentais de outra pessoa com base em informações observáveis, e; 2) de dedução, que envolve a capacidade de integrar as informações contextuais e históricas sobre uma pessoa para entender e predizer o seu comportamento (Sabbagh, 2004). Nesse estudo, foram avaliados 76 pais de sujeitos autistas e 41 pais de crianças saudáveis. Como o objetivo se constituía em avaliar separadamente as habilidades de decodificação e dedução de ToM, foram utilizados quatro instrumentos:1) Eyes Test (27 fotos) (BaronCohen et al., 2001); 2) Faces Test (10 fotos) (Adolphs, Baron-Cohen, \& Tranel, 2002); 3) Unexpected Outcomes Test (UOT) (Dyck, Ferguson, \& Shochet, 2001) e; 4) Hinting Task (quatro histórias) (Corcoran, Mercer, \& Frith, 1995). Os dois primeiros instrumentos visavam a avaliação da habilidade de decodificação e, os dois últimos, a habilidade de dedução. Como resultado, os autores também não encontraram diferenças entre os grupos no que diz respeito à habilidade de decodificação de ToM. Entretanto, os resultados indicaram déficits na habilidade de dedução da teoria da mente em pais de crianças com autismo (Gokcen et al., 2009).

Já citado dentre as pesquisas com irmãos, o estudo de Nydén et al., (2011) utilizou como amostra também os pais de pessoas com TEA. A avaliação da habilidade de Teoria da Mente dos pais também foi realizada por meio do Cartoon Explanation Tasks (non-mental e mental) (Gallagher et al., 2000). O instrumento foi aplicado em pais, mães e irmãos de sujeitos com transtorno autístico. Os achados da pesquisa sugerem a inexistência de déficits específicos na execução da tarefa de teoria da mente nos pais.
Dentre os estudos encontrados, o mais recente avalia conjuntamente crianças com síndrome de Asperger e seus pais, realizando análises em separado de mães e pais (Shimoni, Weizman, Yoran \& Raviv, 2012). Como instrumento de mensuração de ToM foi utilizado o Social Attribution Task (Klin, 2000), uma tarefa nãoverbal que possui como diferencial o fato de ser uma ferramenta que visa minimizar a influência das habilidades verbais do participante. Trata-se de um instrumento no qual é apresentado um filme de formas geométricas se movendo, e o examinando deve, diante do estímulo visual, elaborar narrativas que contenham atribuições sociais de intencionalidade. Os resultados encontrados apontam a presença de déficits na habilidade de ToM em crianças com síndrome de Asperger e suas mães. Não apontam, por outro lado, déficits no grupo de pais avaliado. Diante disto, é sugerida uma contribuição genética mais proeminente das mães, em relação à habilidade de teoria da mente em filhos com síndrome de Asperger.

\section{DISCUSSÃO}

Os estudos revisados têm encontrado resultados inconclusivos, no que se refere à habilidade de teoria da mente em familiares de pessoas com transtornos do espectro do autismo. Em irmãos, há um estudo que aponta déficits de ToM (Dorris et al., 2004), enquanto outros três não encontram diferenças entre os grupos (Nydén et al., 2011; Ozonoff et al., 1993; Shaked et al., 2006). Da mesma forma com pais, existem pesquisas indicativas de déficits de ToM (Baron-Cohen \& Hammer,1997; Baron-Cohen et al., 2006), bem como um estudo que não apresenta evidências de diferenças entre os grupos (Nydén et al., 2011). Além disso, três estudos encontram resultados mais específicos. A partir do estudo de subtipos de características dos pais (FAA) e subtipos de habilidades de Teoria da Mente, Losh e Piven (2007) identificam apenas o subgrupo aloof como acometido por prejuízos de ToM, não encontrando diferenças mais gerais na comparação entre os grupos avaliados. Já Gokcen et al. (2009) encontram resultados que sugerem a inexistência de déficits de habilidade de decodificação de ToM, e, por outro lado, a existência de prejuízos da habilidade de dedução. Por fim, Shimoni et al. (2012) apontam para a existência de prejuízos de ToM em mães, mas não em pais.

Uma hipótese explicativa para a ausência de concordância entre os resultados encontrados remete à complexidade do construto Teoria da Mente e à con- 
sequente dificuldade de avaliação desta dimensão. Desta forma, pode-se supor que os instrumentos utilizados sejam insuficientemente sensíveis para a detecção dos déficits cognitivos subjacentes à Teoria da Mente (Ozonoff et al., 1993, Nýden et al., 2011). Destaca-se, ainda, que fatores psicológicos, sociais e culturais podem funcionar como variáveis intervenientes nos resultados dos instrumentos de avaliação de ToM. Apesar das hipóteses levantadas a respeito da dificuldade de avaliação do construto, são escassas na literatura pesquisas que enfoquem a identificação destes fatores intervenientes, bem como o estudo das ferramentas de testagem de Teoria da Mente. Ainda em relação à avaliação do construto teoria da mente, observa-se que em todas as pesquisas analisadas na presente revisão da literatura foram conduzidas com delineamento observacional transversal. Desta forma, nenhum estudo investigou a estabilidade do construto avaliado.

Outro possível motivo da discordância entre os resultados remete à indiferenciação, nos estudos, entre os subtipos de familiares, no que se refere às características do fenótipo ampliado do autismo (FAA). Pode-se supor, a partir dos resultados encontrados por Losh e Piven (2007), que existam déficits de teoria da mente apenas em familiares de determinados subtipos de FAA, como o aloof.

Uma terceira hipótese explicativa da inconclusibilidade dos resultados se refere à possível necessidade de divisão do construto ToM em duas habilidades: de decodificação e de dedução. Com base nesta ideia, a grande maioria dos estudos já realizados investiga isoladamente apenas uma das dimensões de ToM. Considerando todos os instrumentos utilizados nas pesquisas de Teoria da Mente em familiares de pessoas com TEA, é possível categorizar Second Order Belief Attribution Task, Fox and Grapes Task, Apple-Dog Task, False Belief Task, Strange Stories Task, Unexpected Outcomes Test, Hinting Task, Cartoon Explanation Tasks e Social Attribution Task como formas de investigação da habilidade de dedução, enquanto apenas o Eyes Test e o Faces Test poderiam ser considerados como instrumentos que avaliam a habilidade de decodificação. Esta hipótese ganha força no estudo de Gokcen et al. (2009), que encontra déficits na habilidade de dedução dos pais, e não na habilidade de decodificação.

Cabe, ainda, destacar o pequeno número de estudos encontrados a respeito da teoria da mente em familiares de pessoas com autismo. A escassez de pes- quisas com este enfoque contribui para a inexistência de resultados conclusivos sobre o tema.

Deve-se ressaltar, ainda que todas as pesquisas encontradas na presente revisão da literatura assumem como pressuposto a existência de mecanismos humanos biologicamente determinados que fundamentam as habilidades de teoria de mente. Há, entretanto, muitas críticas à forma de estudo vigente nos estudos empíricos sobre teoria da mente. Segundo Miller (2010) esta forma de abordagem da cognição social entende a pessoa a ser estudada como um objeto cujas propriedades são medidas a fim de descobrir supostos processos cognitivos elementares e seus mecanismos para prever o funcionamento social e a adaptação. Paradiso e Rudrauf (2012) apontam o caráter reducionista do método adotado, que traz consigo o risco de obliterar os aspectos essenciais da cognição social, tendo em vista a sua natureza integrativa e complexa. Tal complexidade se impõe como o desafio fundamental para estudos nos quais o delineamento de pesquisas demanda simplificação. Desta forma, sugere-se que a neurociência cognitiva precisa reapropriar os estudos sobre cognição de suas dimensões sociais, psicológicas e culturais, a fim de definir orientações de pesquisa mais válidas.

\section{CONSIDERAÇÕES FINAIS}

Realizou-se uma revisão sistemática da literatura sobre a teoria da mente em pais e irmãos de pessoas com autismo, de modo a propiciar uma visão abrangente sobre o estado atual deste campo de pesquisa. Uma das limitações do presente estudo se refere à restrição dos idiomas e das bases de dados utilizados na busca dos artigos, uma vez que possivelmente estes critérios geram a exclusão de outros estudos sobre o tema. Apesar disto, buscou-se selecionar idiomas e bases de dados que abarcassem um grande número de artigos, de modo a alcançar uma revisão o mais abrangente possível.

Os estudos sobre teoria da mente em familiares de pessoas com TEA permitem lançar luz a reflexões sobre hipóteses etiológicas do autismo, ao levantar supostos marcadores cognitivos fenotípicos para o transtorno (Gottesman \& Gould, 2003). Além disso, uma delimitação mais clara dos déficits e habilidades típicos em familiares pode gerar reflexões sobre modos específicos de condução da intervenção de Treinamento de Pais para este público, que sejam efetivas para o desenvolvimento da criança. 
Sugere-se, a partir disto, a necessidade de desenvolvimento de mais pesquisas sobre o tema em questão, dada a escassez de estudos disponíveis. Dentre os possíveis enfoques de pesquisa, poderia ser abordado o desenvolvimento de instrumentos de avaliação de ToM mais sensíveis, que permitam acessar operações cognitivas mais elementares a partir de medidas quantitativas de cunho comportamental (Duvall, Lu, Cantor, Constantino, \& Geschwind, 2007), através do controle de variáveis intervenientes, tais como as habilidades de linguagem do examinando. Para isto, tais instrumentos devem ser eles mesmos alvo de estudo, de modo a garantir que a ferramenta de pesquisa apresente propriedades psicométricas adequadas para a medição do construto Teoria da Mente.

Outro caminho para estudos futuros consiste na replicação e ampliação de estudos como o de Losh e Piven (2007), que avaliem ToM diferenciando os três subgrupos de características do fenótipo ampliado do autismo (FAA): personalidade rígida, personalidede aloof (distante) e déficits de linguagem pragmática. Além disso, o desenvolvimento de mais estudos que avaliem ToM diferenciando habilidade de decodificação e de dedução, como o de Gokcen et al. (2009), permitirão esclarecer se teoria da mente se trata de um construto unidimensional, ou se a divisão entre duas subcategorias da habilidade traz contribuições para o tema em questão. Além disso, a realização de análises em separado dos resultados de ToM de pais e mães, tal como realizado por Shimoni et al. (2012), podem contribuir para o esclarecimento sobre a contribuição de cada um dos pais no déficit de teoria da mente de seu filho.

Por fim, uma outra alternativa de pesquisa sobre ToM seria a condução de estudos naturalísticos, que viabilizem uma análise da teoria da mente em contextos de interação social. Este delineamento de pesquisa poderia evidenciar os aspectos sociais, culturais e psicológicos envolvidos na capacidade de atribuir estados mentais para si mesmo e para os outros, trazendo uma visão mais abrangente sobre os fatores que interferem na ocorrência de déficits nesta habilidade.

\section{REFERÊNCIAS}

Adolphs, R., Baron-Cohen, S., \& Tranel, D. (2002). Impaired recognition of social emotions following amygdala damage. Journal of Cognitive Neuroscience, 14, 1264- 1274.

Associação Americana de Psiquiatria (2002). Manual diagnóstico e estatístico de transtornos mentais (DSM IV). Tradução de Cláudia Dornelles. $4^{\mathrm{a}}$ ed. Porto Alegre: Artes Médicas.
Baron-Cohen S. (1989). The autistic child's theory of mind: A case of specific developmental delay. Journal of Child Psychology and Psychiatry, 30, 285-297.

Baron-Cohen, S. \& Hammer, J. (1997). Parents of children with Asperger syndrome: what is the cognitive phenotype? Journal of Cognitive Neuroscience, 9, 4, 548-554.

Baron-Cohen, S., Jolliffe, T., Mortimore, C., \& Robertson, M. (1997). Another advanced test of theory of mind: Evidence from very high functioning adults with autism or Asperger Syndrome. Journal of Child Psychology and Psychiatry, 38, 813-822.

Baron-Cohen, S., Leslie, A. M., \& Frith, U. (1985). 'Does the Autistic Child Have a "Theory of Mind"?' Cognition, 21, 3746.

Baron-Cohen, S., Ring, H., Chitnis, X., Wheelwright, S., Gregory, L., Williams, S., Brammer, M. \& Bullmore, E. (2006). fMRI of parents of children with Asperger syndrome: A pilot study. Journal of Brain Cognition, 61, 122-130.

Baron-Cohen, S., Wheelwright, S., Hill, J., Raste, Y., \& Plumb, I. (2001). The 'Reading the Mind in the eyes' test revised version: A study with normal adults, and adults with Asperger Syndrome or High-Functioning autism. Journal of Child Psychology and Psychiatry, 42, 241-252.

Baron-Cohen, S., Wheelwright, S., Stone V., \& Rutherford, M. (1999). A mathematician, a physicist, and a computer scientist with Asperger Syndrome: Performance on folk psychology and folk physics tests. Neurocase, 5, 475-483.

Best, C. S, Moffat V.J., Power M.J., Owens D.G., \& Johnstone E.C. (2008). The boundaries of the cognitive phenotype of autism: theory of mind, central coherence and ambiguous figure perception in young people with autistic traits. Journal of Autism and Developmental Disorders, 38, 840-847.

Bolte, S., \& Poustka, F. (2006). The broader cognitive phenotype of autism in parents: How specific is the tendency for local processing and executive dysfunction? Autism Research, 47, $639-645$.

Corcoran, R., Mercer, G., \& Frith, C.D. (1995). Schizophrenia, symptomatology and social inference: investigating theory of mind in people with schizophrenia. Schizophrenia Research, 17, 5-13.

De Vignemont, F., \& Singer, T. (2006). The empathic brain: How, when, and why? Trends in Cognitive Sciences, 10, 435-441.

Dennet, D. C., (1978). Beliefs about beliefs. The Behavioral and Brain Sciences, 1, 568-570.

Dorris, L., Espie, C. A., Knott, F., \& Salt, J. (2004). Mind-reading difficulties in the non-affected siblings of people with Asperger's syndrome: Evidence for a genetic influence in the abnormal development of a specific cognitive domain. Journal of Child Psychology and Psychiatry, 45, 412-418.

Duvall, J. A., Lu, A., Cantor, R. D., Constantino, J. N., \& Geschwind, D. H. (2007). A quantitative trait locus analysis of social responsiveness in multiple autism families. American Journal of Psychiatry, 164, 656-662. 
Dyck, M. J., Ferguson, K., \& Shochet, I. M. (2001). Do autism spectrum disorders differ from each other and from nonspectrum disorders on emotion recognition tests? European Child and Adolescent Psychiatry, 10, 105-116.

Flavell, J. H., Botkin, P. T., Fry, C. L., Wright, J. W. \& Jarvis, P. E. (1968). The development of role-taking and communication skills in children. Nova York: Wiley.

Ford, R. M., Lobao, S. N., Macaulay, C., \& Herdman, L. M. (2011). Empathy, theory of mind, and individual differences in the appropriation bias among 4- and 5-year-olds. Journal of Experimental Child Psychology, 110, 626-646.

Frith, U., \& Frith, C. D. (2003). Development and neurophysiology of mentalizing. Philosophical Transactions of the Royal Society of London B: Biological Sciences, 358, 459-473.

Gallagher, H., Happé, F., Brunswick, N., Fletcher, P., Frith, U., \& Frith, C. (2000). Reading in the mind cartoons and stories: An fMRI study of "theory of mind" in verbal and non-verbal tasks. Neuropsychologia, 38, 11-21.

Gallese, V. (2003). The roots of empathy: The shared manifold hypothesis and the neural basis of intersubjectivity. Psychopathology, 36, 171-180.

Gokcen, S., Bora, E., Erermis S., Kesikci H., \& Aydin, C. (2009). Theory of mind and verbal working memory deficits in parents of autistic children. Psychiatry Research, 166, 46-53.

Gottesman, I. I., \& Gould, T. D. (2003). The endophenotype concept in psychiatry: Etymology and strategic intentions. Journal of American Psychiatry, 160, 636-645.

Happé, F. (1994). 'An Advanced Test of Theory of Mind: Understanding of Story Characters' Thoughts and Feelings by Able Autistic, Mentally Handicapped, and Normal Children and Adults', Journal of Autism and Developmental Disorders, 24, 129-53.

Hurley, R. S. E., Losh M., Parlier, Reznick, M., J. S., Piven, J. (2007). The Broad Autism Phenotype Questionnaire. J Autism Dev Disord, 37, 1679-1690.

Jou, G. I. De, \& Sperb, T. M. (1999). Teoria da Mente: Diferentes Abordagens. Psicologia Reflexão e Crítica, 17, 167-176.

Kamp-Becker, I. et al. (2009). Dimensional structure of the autism phenotype: relations between early development and current presentation. Journal of Autism and Developmental Disorders, $39,4,557-571$.

Kelemenova S., \& Ostatnikova D. (2009). Neuroendocrine pathways altered in autism. Special role of reelin. Neuro Endocrinol Letters, 30, 4, 429-36.

Kim, Y. S., Leventhal, B. L., Koh, Y-J, Fombonne, E., Laska , E., Lim, E-C, ... \& Grinker, R. R. (2011). Prevalence of Autism Spectrum Disorders in a Total Population Sample. American Journal of Psychiatry, 168, 904-912.

Klin, A. (2000). Attributing social meaning to ambiguous visual stimuli in higher functioning autism and Asperger syndrome: the Social Attribution Task. Journal of Child Psychology and Psychiatry, 41, 831-846.
Lamm, C., Batson, C. D., \& Decety, J. (2007). The neural substrate of human empathy: Effects of perspective-taking and cognitive appraisal. Journal of Cognitive Neuroscience, 19, 4258.

Losh, M., \& Piven, J. (2007). Social-cognition and the broad autism phenotype: indentigying genetically meaningful phenotypes. Journal of Child Psychology and Psychiatry, 48, 1, 105112.

Mercadante M. T., Gaag R. J. V., \& Schwartzman J. S. (2006). Transtornos invasivos do desenvolvimento não-autísticos: síndrome de Rett, transtorno desintegrativo da infância e transtornos invasivos do desenvolvimento sem outra especificação. Revista Brasileira de Psiquiatria, 28, 12-20.

Miller GA. Mistreating psychology in the decades of the brain. Perspect Psychol Sci. 2010;5:716-743.

Nýden, A., Hagberg, B., Goussé, V., \& Rastam, M. (2011). A cognitive endophenotype of autism in families with multiple incidence. Research in Autism Spectrum Disorders, 5, 191-200.

Ozonoff, S., Rogers, S. J., Farnham, J. M., \& Pennington B. F. (1993) Can standard measures identify subclinical markers of autism? Journal of Autism \& Developmental Disorders, 23, 429-441.

Paradiso, S. \& Rudrauf, D. (2012). The self in social cognitive neuroscience. Dialogues in Clinical Neuroscience, 14, 1, 6575 .

Pellicano E., Maybery M., Durkin K., \& Maley A. (2006). Multiple cognitive capabilities/deficits in children with an autism spectrum disorder: "weak" central coherence and its relationship to theory of mind and executive control. Development and Psychopathology, 18, 77-98.

Piven, J., Wzorek, M., Landa, R., Lainhart, J., Bolton, P., Chase, G., \& Folstein, S. (1994). Personality characteristics of the parents of autistic individuals. Psychological Medicine, 24, 783795.

Premack, D., \& Woodruff, G. (1978). Does the chimpanzee have a theory of mind? Behavioural and Brain Science, 1, 515-526.

Roazzi, A., \& Santana, S. de M. (2008). Teoria da mente e estados mentais de primeira e segunda ordem. Psicologia: Reflexão $e$ Crítica, 21, 437-445.

Sabbagh, M.A. (2004). Understanding the orbitofrontal contributions to theory of mind reasoning: implications for autism. Brain and Cognition, 55, 209-219.

Scheeren, A., \& Stauder, J. (2008). Broader autism phenotype in parents of autistic children: Reality or myth? Journal of Autism and Developmental Disorder, 38, 276-287.

Shaked, M., Gamliel, I., \& Yirmiya, N. (2006). Theory of mind in young siblings of children with autism. Autism, 10, 173-187.

Shamay-Tsoory, S. G., Aharon-Peretz, J., \& Perry, D. (2009). Two systems for empathy: A double dissociation between emotional and cognitive empathy in inferior frontal gyrus versus ventromedial prefrontal lesions. Brain, 132, 617-627. 
Shimoni, H. N., Weizman, A., Yoran, R. H., \& Raviv, A. (2012). Theory of mind, severity of autistic symptoms and parental correlates in children and adolescents with Asperger syndrome. Psychiatry Research, 197, 85-89.

Singer, T. (2006). The neuronal basis and ontogeny of empathy and mind reading: Review of literature and implications for future research. Neuroscience and Biobehavioral Reviews, 30, $855-863$

Wellman, H. M., Cross, D., \& Watson, J. (2001). Meta-analysis of theory of mind development: The truth about false belief. Child Development, 72, 655-684.
Wimmer, H., \& Perner, J. (1983). Beliefs about beliefs: Representation and constraining function of wrong beliefs in young children's understanding of deception. Cognition, 13, 103-28.

Recebido em 29/05/2012 Primeira Decisão Editorial em 17/12/2012 Segunda Decisão Editorial em 21/02/2013 Aceito em 09/04/2013 